\title{
Zur weichselzeitlichen Entwicklungsgeschichte der unteren Saar (Rheinisches Schiefergebirge) -
}

\author{
Eine geomorphologische, pedologische und palynologische Untersuchung * \\ Manfred J. Müller, Dietmar Schröder, Brigitte Urban \& Ludwig Zöller **)
}

Morphogenesis, River Saar Low Terrace, meander, glacial deposit, Saar Canal, flood deposit, paleosol, alluvial soil, solifluction, Weichselian, Bölling Interstadial, pit section, pollen analysis, C 14 dating, size distribution, chemical composition.

Rhenish Schiefergebirge, Lower Saar River, Rhineland Palatinate. TK 25: Nr. 6305

Kurzfassung: Durch den Bau des Saarkanals im Zuge des Umlauftals Ayl-WawernBiebelhausen entstand ein fast $2 \mathrm{~km}$ langer Aufschluß, der über die Entwicklung der Saar von der letzten Kaltzeit bis heute neue Erkenntnisse brachte. An zwei günstigen Stellen sind mehr als $12 \mathrm{~m}$ mächtige Sande erhalten geblieben, die aufgrund des Fehlens der vulkanischen Eifelassoziation im Schwermineralspektrum und wegen eindeutiger Verzahnung mit Solifluktionsschutt ins Weichselglazial gestellt werden müssen. Diese Sande liegen auf dem etwa $2 \mathrm{~m}$ mächtigen Basisschotter, der ebenfalls als letztglaziale Ablagerung gedeutet werden muß. In einem Stillwassersediment (schluffiger Ton), das auf den Mäanderdurchbruch hinweist, wurde ein Stück Kiefernholz gefunden, das ein Alter von $15800 \pm 300$ Jahre aufweist.

In den als Hochwasserablagerungen gedeuteten Sanden konnten 3 verschiedene, recht gut ausgebildete Paläoböden ausgemacht werden.

Mit Hilfe der Pollenanalyse und zweier ${ }^{14} \mathrm{C}$-Daten konnten die Böden zeitlich bestimmt werden. Der älteste ist mit groß3er Wahrscheinlichkeit während des Amersfoort-Interstadials ausgebildet worden. Der mittlere Boden ist, im Gegensatz zu den beiden anderen, in einer trockeneren, warmen Phase des Weichselfrühglazials entstanden und wird deswegen ins Brørup-Interstadial gestellt. Sein wesentliches Merkmal ist das Vorkommen von Haselpollen. Der oberste Boden muß ins Spätglazial datiert werden. $\mathrm{Da}$ weder im Boden noch in der darüberliegenden Fließerde die Schwermineralien der Eifelassoziation (Laacher Vulkan) gefunden wurden, ist dieser Boden älter als das Alleröd. Er wird als Bildung im Bölling-Interstadial aufgefaßt.

Aufgrund dieser Ergebnisse lassen sich über die Entwicklungsgeschichte der unteren Saar sehr viel detailliertere Aussagen machen als das bisher der Fall war.

\section{[The Evolutional History of the River Saar during the Weichsel Glacial Period - a Geo- morphological, Pedological and Pollenanalytical Investigation]}

A bstract: Because of the construction of the Saar Canal an opening of nearly $2 \mathrm{~km}$ length resulted along the cut-off meander of Ayl-Wawern-Biebelhausen. This opening permitted new perceptions with regard to the evolutional history of the River Saar from the last glacial period until today. At two favourable locations sands of more than $12 \mathrm{~m}$ thickness have been preserved. Due to the absence of any volcanic heavy minerals from the Eifel and due to a very clear interlocking with solifluction debris these sands must be placed into the Weichselian glacial period. The sands lie on top of gravel of about $2 \mathrm{~m}$ thickness which should also be interpreted as belonging to the last glaciation. Within a stillwater sediment (silty clay), which points out a meander cut-off, a piece of pine wood was found that shows an age of $15800 \pm 300$ years.

*) Die geomorphologischen Untersuchungen wurden mit Unterstützung der Deutschen Forschungsgemeinschaft durchgeführt. Dafür sei ihr herzlich gedankt.

**) Anschriften der Verfasser: Dr. M. J. M ülle r, Prof. Dr. D. S ch rö d e r, L. Z öll e r, M. A., Universität Trier, FB III, Geographie/Geowissenschaften, Postfach 3825, D-5500 Trier. Dr. B. Urban-Küttel, Institut für Bodenkunde der Universität Bonn, Nußallee 13, D-5300 Bonn. 


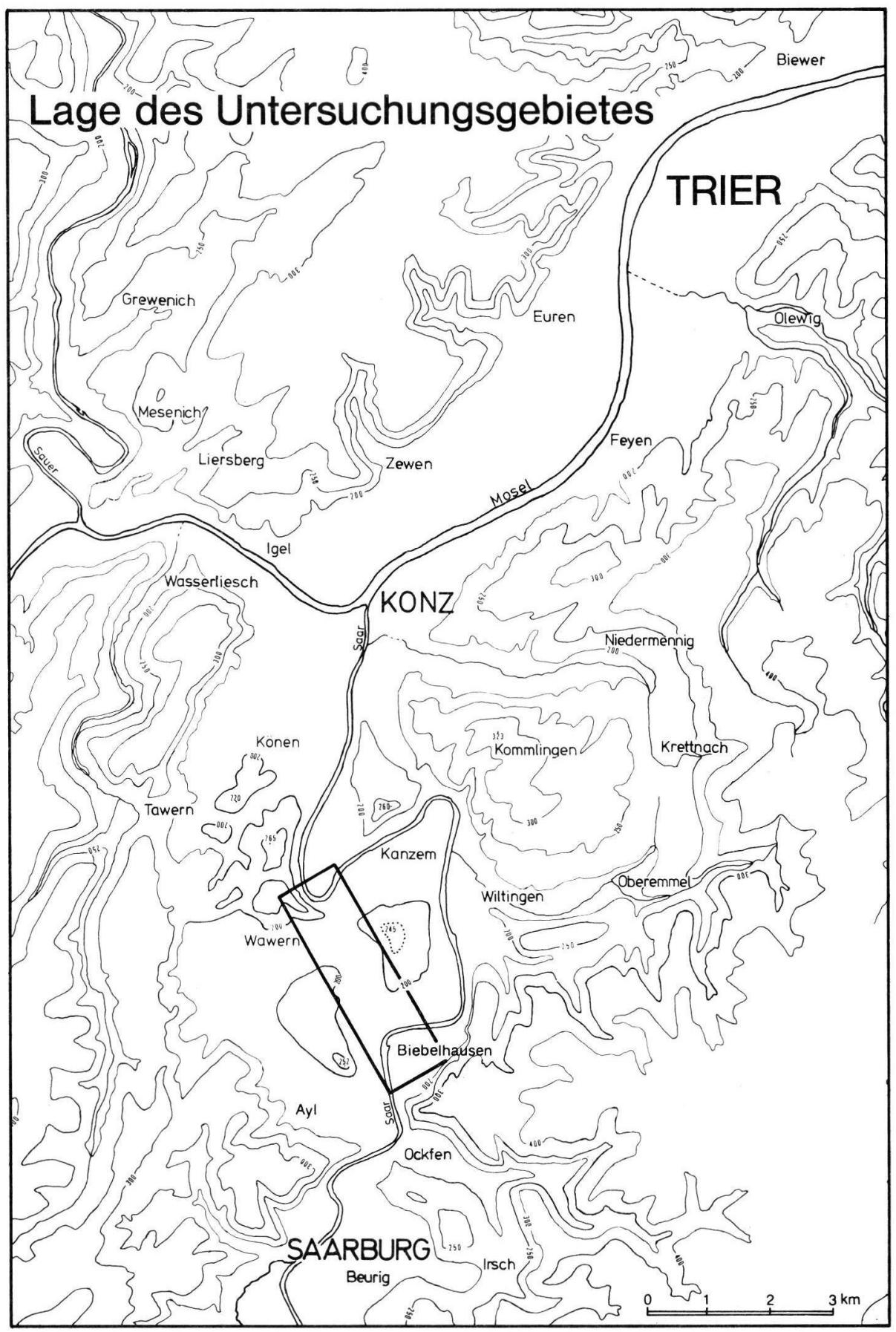

Abb. 1: Lage des Untersuchungsgebietes. 
Within the sands, which are being interpreted as flood deposits, three different and welldeveloped paleosoils were detected.

Using the pollen analysis and two ${ }^{14} \mathrm{C}$-dates the soils could be placed into the proper temporal perspective. In all likelyhood the oldest soil developed during the Amersfoort interstadial epoch. The middle one, in contrast to the two others, was formed during a more arid and warmer phase of the early Weichsel glacial period. Therefore, it should be placed into the Brørup interstadial. Its significant feature is the presence of hazel pollen. The uppermost soil must be placed into the late glacial period. Since nowhere, neither in the soil itself nor in the solifluction debris on top of it, the heavy minerals of the Eifel association (Laach Volcano, 11000 years BP.) could be found, this soil must be older than the Alleröd. It is interpreted as having been developed during the Bölling interstadial.

Because of the aforementioned results more detailed statements can now be made on the evolutional history of the lower Saar than before.

\section{Einleitung}

Der Ausbau der Saar zu einem Schiffahrtsweg bot die einmalige Gelegenheit, neue Untersuchungen über die weichselzeitliche und holozäne Entwicklung der unteren Saar durchzuführen. Zwar sind die Laufverlegungen unterhalb von Saarburg mehrfach untersucht worden, so z. B. von Grebe (1886), Mathias (1936 u. 1952), und Müller (1976), es konnten bisher aber nicht alle Probleme befriedigt gelöst werden.

Die zahlreichen Tiefbohrungen, die wegen der Baumaßnahmen immer bis auf das Anstehende abgeteuft worden sind, vor allem aber der Durchstich zwischen Hamm und Biebelhausen (Abb. 1), schufen sehr gute Voraussetzungen. Der fast $2 \mathrm{~km}$ lange Durchstich verläuft im Zuge des Ayl-Wawern-Biebelhausener Mäanders (Abb. 2), der während des Weichselglazials aufgrund des Durchbruchs zwischen Biebelhausen und Schoden abgeschnitten worden ist. Der Aufschluß konnte abschnittsweise, entsprechend dem Baufortschritt, aufgenommen werden, so daß detaillierte stratigraphische, pedologische und palynologische Untersuchungen möglich waren.

\section{Beobachtungen und Ergebnisse}

\subsection{Aufbau und Altersstellung der Sedimente}

Die Flußsedimente sind an keiner Stelle dieses Talabschnittes in ihrer gesamten Abfolge übereinander erhalten. Das erschwerte die Rekonstruktion der talgestaltenden Prozesse. Überraschenderweise liegen am Talrand in einer vor Abtragung offensichtlich geschützten Position mehr als $12 \mathrm{~m}$ mächtige schräggeschichtete Sande. Sie fallen zunächst steil mit $15^{\circ}-20^{\circ}$, dann sanft mit $5^{\circ}-10^{\circ}$ zur Flußmitte hin ein (Abb. 3). Am Rand sind sie mit Solifluktionsschutt verzahnt und werden von einer Fließerde überlagert.

In diesen Sanden wurden 3 fossile Böden festgestellt (s. Profilbeschreibung). Eine besondere Rolle spielt der mittlere Boden in einer Tiefe von 8,40-9,90 m unter Geländeoberkante. Er fiel im Gelände durch seine dunkle Färbung und sein gut ausgebildetes Gefüge auf und wurde als Auenbraunerde aus Hochflutlehm angesprochen. Im oberen Teil ist das Gefüge plattig, im unteren Teil subpolyedrisch. Regenwurmgänge treten nur im unteren Teil auf. Im Dünnschliff ist ein gut ausgebildetes und sehr dunkles Plasma zu erkennen. Die Analysen ergaben gegenüber den beiden anderen fossilen Böden vor allem Unterschiede im C-Gehalt, der hier mit 0,50\% recht hoch liegt (Tab. 3).

Der relativ hohe Humusgehalt, die große Mächtigkeit dieses Horizontes $(8,40 \mathrm{~m}$ und $9,25 \mathrm{~m}$ ) und das plattige Gefüge in $8,40-8,80 \mathrm{~m}$ sprechen für eine solifluidale Überlagerung des ursprünglichen A-Horizontes. 


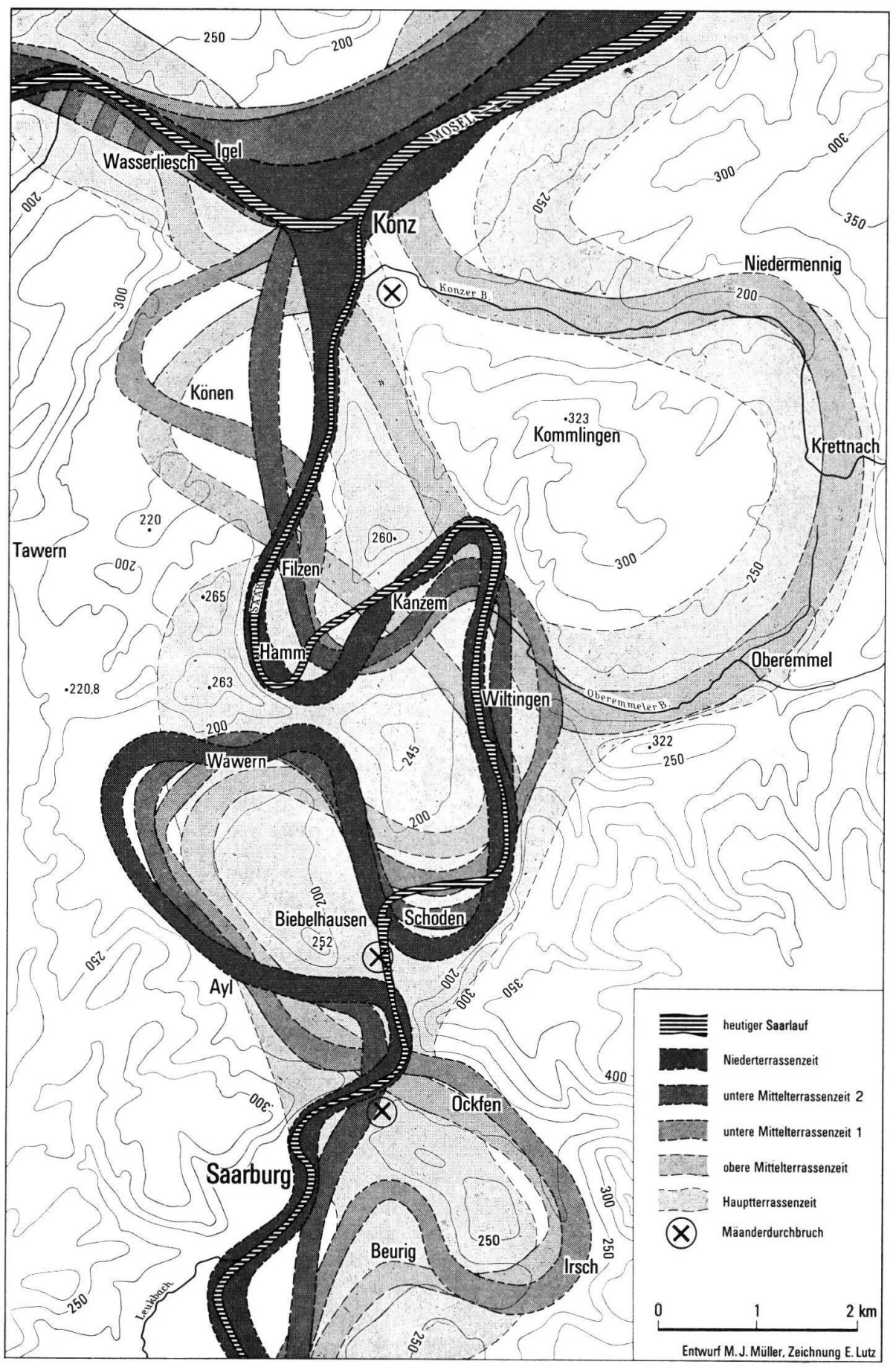

Abb. 2: Laufverlegungen an der unteren Saar. 

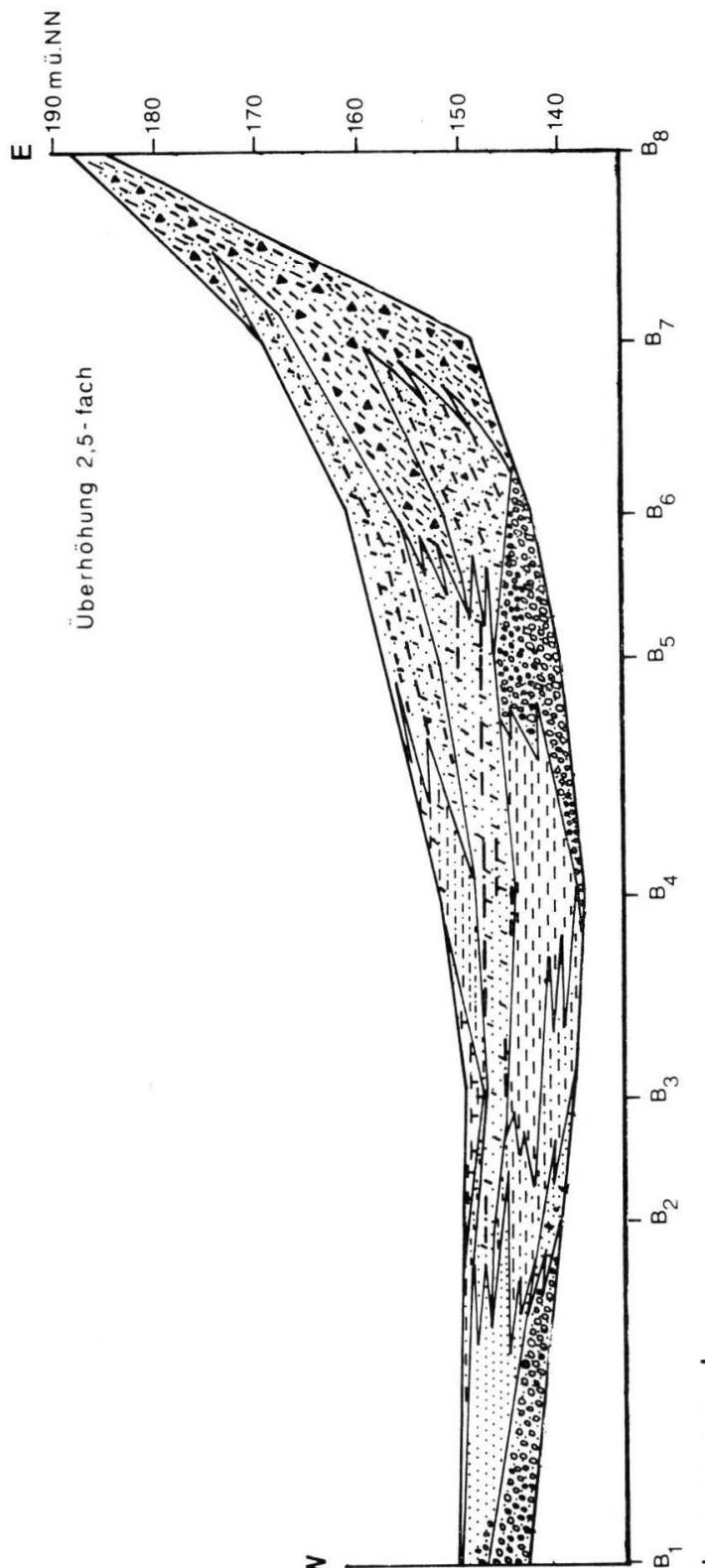

Abb. 3: Saarmäander Ayl-Wawern-Biebelhausen, Profil Weingut Greif.

Der obere fossile Boden (6,00-8,40 m unter Geländeoberkante) hat sich auf einem mächtigen Hochflutsand entwickelt. Er war im Gelände an seiner leicht humusgetönten Farbe, dem subpolyedrisch ausgebildeten Gefüge und zahlreichen Regenwurmgängen zu erkennen. Im unteren Abschnitt treten 5-10 cm mächtige dunklere, honigsandartig (kol- 
Tab. 1: Korngrößenverteilung in den Böden

\begin{tabular}{lcccc} 
& & \multicolumn{3}{c}{ Korngröße in $\mu \mathrm{m}$ in $\%$ Feinboden } \\
\cline { 3 - 5 } Tiefe & Horizont & Ton & Schluff & Sand \\
\cline { 3 - 5 }$(\mathrm{cm})$ & & $<2$ & $2-63$ & $63-2000$ \\
\hline
\end{tabular}

rezenter Boden

$\begin{array}{rlrrr}0-20 & \mathrm{~A}_{\mathrm{h}} & 16,60 & 32,00 & 51,40 \\ 20-50 & \mathrm{~A}_{1} \mathrm{~B}_{\mathrm{v}} & 19,85 & 38,40 & 41,75 \\ 50 \text { - } 85 & \mathrm{~A}_{\mathrm{l}} \mathrm{S}_{\mathrm{w}} & 18,10 & 40,60 & 41,30 \\ 85-110 & \text { II } \mathrm{B}_{\mathrm{t} 1} \mathrm{Sd} & 16,75 & 28,10 & 55,15 \\ 110-160 & \text { II } \mathrm{B}_{\mathrm{t} 2} \mathrm{Sd} & 19,10 & 27,65 & 53,25\end{array}$

oberer fossiler Boden

$\begin{array}{lrrrr}600-625 & \mathrm{f} \mathrm{A}_{\mathrm{h}} \mathrm{M}_{1} & 16,80 & 26,85 & 56,35 \\ 625-650 & \mathrm{M}_{2} & 15,70 & 24,00 & 60,30 \\ 780-790 & & 6,40 & 5,40 & 88,20 \\ 790-800 & & 19,05 & 6,00 & 74,95\end{array}$

mittlerer fossiler Boden

\begin{tabular}{|c|c|c|c|c|}
\hline $\begin{array}{l}840-880 \\
880-925 \\
925-960 \\
960-990\end{array}$ & $f \mathrm{~A}_{\mathrm{h}} \underset{\mathrm{B}_{\mathrm{v}^{2}}}{\mathrm{~B}_{\mathrm{v}^{1}}}$ & $\begin{array}{r}16,90 \\
17,90 \\
8,80 \\
8,30\end{array}$ & $\begin{array}{l}17,25 \\
16,20 \\
17,30 \\
13,50\end{array}$ & $\begin{array}{l}65,80 \\
65,90 \\
73,90 \\
78,20\end{array}$ \\
\hline \multicolumn{5}{|c|}{ unterer fossiler Boden } \\
\hline $\begin{array}{r}990-1020 \\
1020-1115 \\
1115-1215\end{array}$ & $\mathrm{~B}_{\mathrm{t}}$ & $\begin{array}{r}11,88 \\
3,73 \\
9,25\end{array}$ & $\begin{array}{r}16,92 \\
9,22 \\
5,17\end{array}$ & $\begin{array}{l}71,20 \\
87,05 \\
85,58\end{array}$ \\
\hline
\end{tabular}

loidale Umhüllungen der Sandkörner) mit Feinton und Eisen angereicherte Bänder auf (Tab. 1; 7,90-8,00 m). Die bodenchemischen Daten, speziell die mit der Tiefe abnehmenden $\mathrm{C}$ - und $\mathrm{P}_{2} \mathrm{O}_{5}$-Gehalte, bestätigen die aufgrund des Geländebefundes vorgenommene Identifikation dieses Bodens als Allochthonen Braunen Auenboden mit schwacher Tondurchschlämmung im unteren Profilteil und mit geringfügig verkürztem $A_{h}$-Horizont.

Der untere Boden (9,90-11,15 m unter Geländeoberkante) muß als Rest einer AuenParabraunerde angesprochen werden. $A_{h^{-}}$und $A_{1}$-Horizont fehlen. Nur ein etwa $30 \mathrm{~cm}$ mächtiger $\mathrm{B}_{\mathrm{t}}$-Horizont mit polyedrischem Gefüge ist erhalten. Unterhalb des kompakten $\mathrm{B}_{\mathrm{t}}$-Horizontes befinden sich in sandigen Partien Eisen-Ton-Bändchen bzw. -Nester, die im oberen fossilen Boden Honigsandcharakter haben ${ }^{1}$ ).

Ein Vergleich des bodengenetischen Zustandes der drei fossilen Böden zeigt, daß die Entwicklung im untersten Boden am weitesten fortgeschritten ist. Hier hat eine Lessivierung stattgefunden, die in den verwitterten Hochflutlehmen bei gegebenen kolloidchemischen Voraussetzungen allerdings in relativ kurzen Zeiträumen ablaufen kann (Schröder 1979, Stephan 1980). Eine intensive chemische Verwitterung ist, wie die hier nicht mitgeteilten Gesamtgehalte verschiedener Elemente und der Anteil von pedogenem Eisen am Gesamteisen erkennen lassen, jedoch nicht erfolgt. Insgesamt deutet die Ausbildung dieses Bodens auf kühlfeuchte Entstehungsbedingungen (schwache Hydromorphie).

1) Für die mikromorphologische Ansprache der Böden danken wir Herrn Dr. Stephan, Bonn. 
Im mittleren Boden ist die Entwicklung nicht über ein Braunerdestadium hinausgegangen. Die dunkle Farbe und der relativ hohe C-Gehalt lassen auf ein trockenes, wärmeres Klima schließen. In einer anschließenden Kaltphase wurden die obersten $40 \mathrm{~cm}$ durch Solifluktion aufgelagert (Frostplattung, Zerstörung der Wurmgänge). Der obere Boden befindet sich im Ubergang von einem Allochthonen Braunen Auenboden zu einer Parabraunerde. Hierauf deuten die Homogenität im oberen Profilteil und die beginnende Bildung von Eisen- und Ton-Bändern in 1-2 m Tiefe. Schwache Hydromorphie zeigt an, daß bei der Entstehung dieses Bodens ebenfalls feuchtere und kühlere Klimabedingungen geherrscht haben als bei der Entstehung des mittleren Bodens.

Die scheinbar ungestörte Abfolge der Böden legte zunächst den Schluß nahe, es handle sich um 3 Böden, die über einen kurzen Zeitraum hinweg im Spätglazial entstanden sind. Die sedimentpetrographischen, pedologischen und palynologischen Analysenergebnisse widerlegen diese Auffassung jedoch.

Folgende Ubberlegungen lassen auf ein höheres Alter der beiden untersten Böden schließen:

1. Der obere Boden ist vor dem Alleröd entstanden, denn es finden sich in ihm keine Schwermineralien der sogenannten Eifelassoziationen, die den letzten Ausbruch des LaacherVulkans repräsentiert und in der Umgebung von Trier überall in post-allerödzeitlichen Bildungen nachweisbar ist (ZöLLER 1980). Auch in der darüberliegenden Fließerde findet sich die Eifelassoziation nicht. Sie muß also älter als das Alleröd sein und wird in die Ältere Dryas gestellt.

Dieser Boden, dessen Pollenführung für eine quantitative und qualitative Aussage zu gering war, müßte demnach mindestens B öll ing-Alter haben. Bölling- und Allerödböden aus Löß sind unter vergleichbaren Klimabedingungen nur zu Pararendzinen oder Braunerde-Pararendzinen ausgebildet. Das belegen die Pararendzinen und schwach entwickelten Braunerden aus Löß unter dem allerödzeitlichen Bims des Neuwieder Beckens. Die in diesem (Bölling-) Boden abgelaufene Tonverlagerung widerspricht nicht den bisherigen Befunden böllingzeitlicher Lößböden, denn in kalkfreien und kalkarmen Hochflutlehmen kann Tonverlagerung viel schneller einsetzen als im kalkreichen Löß.

2. Der mittlere Boden muß wesentlich älter sein als der obere. Das plattige Gefüge im oberen Teil und die fehlenden Regenwurmgänge weisen auf kaltzeitliche Umlagerung hin. Er scheint andererseits im Unterschied zu den anderen Böden unter warm-trockenen Bedingungen entstanden zu sein. Darauf deutet der mächtige humose Horizont hin, vor allem aber das Pollenspektrum des $f_{h}-$ Horizontes (Abb. 5). Es fällt der hohe Anteil wärmeliebender Taxa auf. Die Hasel erreicht annähernd Werte bis $40 \%$. Auch Eichenmischwaldelemente kommen vor (Linde, Esche, zusammen 10\%, Eichenpollen fehlt). Ein solches Spektrum würde für einen warmzeitlichen Vegetationstyp sprechen, der zudem typisch für ein relativ feuchtes Milieu ist, worauf die Erlenanteile von $28 \%$ hinweisen. $\mathrm{Da}$ aus geomorphologischer Sicht eine Zuordnung zum letzten Interglazial nicht in Frage kommt - der Boden ist auf den Ablagerungen der weichselzeitlichen Niederterrasse ausgebildet - wäre eine Zuordnung zu einem Frühweichselinterstadial $\mathrm{zu}$ diskutieren. Am klimatisch günstigsten scheint das $\mathrm{B}$ rø r u p - Interstadial gewesen zu sein. Eine genaue biostratigraphische Zuordnung ist jedoch aus pollenanalytischer Sicht nicht möglich, da auch in diesem mittleren Boden der Gesamtsporomorphengehalt sehr gering ist und sich die Aussagen nur auf eine Probe beziehen. 
3. Die schwache Fe-Mn-Fleckung des unteren fossilen Bodens und die Ausbildung eines tonangereicherten Horizontes stehen zweifellos nicht in Einklang mit den trockenen und kühlen Klimaverhältnissen des Weichsel-Frühglazials. Fe-Mn-Flekkung als Folge von Staunässe kommt nicht in Betracht, weil der hohe Grobporenanteil und die gute Luftdurchlässigkeit (Tab. 2) Wasserstau ausschließen. Die Ursache der

Tab. 2: Bodenphysikalische Eigenschaften der Böden

\begin{tabular}{|c|c|c|c|c|c|}
\hline $\begin{array}{l}\text { Tiefe } \\
(\mathrm{cm})\end{array}$ & Horizont & $\begin{array}{c}\text { Porenvolumen } \\
(\% / 0)\end{array}$ & $\begin{array}{c}\text { Poren }(\%) \\
>50\end{array}$ & $\begin{array}{l}\text { Trodkenraum- } \\
\text { gewicht } \\
\left(\mathrm{g} / \mathrm{cm}^{3}\right)\end{array}$ & $\begin{array}{l}\text { Luftdurch- } \\
\text { lässigkeit } \\
\mu^{2}\end{array}$ \\
\hline \multicolumn{6}{|c|}{ rezenter Boden } \\
\hline $85-100$ & II $B_{t 1} \mathrm{Sd}$ & 32,93 & 4,63 & 1,77 & 2,18 \\
\hline \multicolumn{6}{|c|}{ rezenter Boden } \\
\hline $110-160$ & II $\mathrm{B}_{\mathrm{t}^{2}} \mathrm{SdII}$ & 35,20 & 7,98 & 1,77 & 7,10 \\
\hline \multicolumn{6}{|c|}{ oberer fossiler Boden } \\
\hline $625-650$ & $\mathrm{f} \mathrm{M}_{2}$ & 41,43 & 14,68 & 1,61 & 7,10 \\
\hline \multicolumn{6}{|c|}{ mittlerer fossiler Boden } \\
\hline $880-925$ & $\mathrm{~B}_{\mathrm{v}^{2}}$ & 45,15 & 18,48 & 1,53 & 9,10 \\
\hline \multicolumn{6}{|c|}{ unterer fossiler Boden } \\
\hline $990-1020$ & $\mathrm{~B}_{\mathrm{t}}$ & 46,48 & 23,70 & 1,59 & 11.08 \\
\hline
\end{tabular}

schwach ausgeprägten Hydromorphie muß deshalb ihren Grund in den besonderen hydrologischen Bedingungen der Talaue haben. Die Pollenführung ist äußerst schwach. Für eine stratigraphische Einordnung käme das Amersfoort-Interstadial in Frage.

Die Abweichung der Auenbodenausbildung von der für diesen Zeitabschnitt bekannten terrestrischen Bodenbildung ist nicht ungewöhnlich (RICKen \& MEYER 1982). Man kann davon ausgehen, daß neben den zonalen Böden auch davon abweichende azonale entstanden sind.

Der Ablauf von Erosion und Akkumulation während des Weichselglazials im Gebiet der unteren Saar ist aufgrund der dargestellten Ergebnisse komplizierter als bisher vermutet.

$\mathrm{Da}$ an keiner Stelle des Aufschlusses die gesamte Abfolge der Sedimente erhalten war, ergab sich die Notwendigkeit, mit Hilfe verschiedener Einzelprofile den Gesamtaufbau der Sedimente zu rekonstruieren (Abb. 3 u. 4).

An der Basis liegt über dem devonischen Schiefer ein $1 \mathrm{~m}$ bis $2 \mathrm{~m}$ mächtiger Grobschotter. In der Talmitte schließt sich darüber ein etwa 2,5 m mächtiger Feinschotter an. Er konnte nirgends unter den randlichen Sanden beobachtet werden. Es wird daher angenommen, daß er jünger ist als die etwa $6 \mathrm{~m}$ mächtigen Sande, in denen der untere und der mittlere Boden liegen. Diese Sande überlagern deutlich den Grobschotter. In die Feinschotter sind an verschiedenen Stellen in Kolken geschichtete schluffige Tone eingelagert. Sie erreichen eine Mächtigkeit von fast $6 \mathrm{~m}$. Die Farbe wechselt zwischen dunkelgrau und rotbraun. Im unteren Teil treten in den Tonen einzelne Schichten schluffigen Sandes, im oberen Teil dünne Lagen kleiner Schieferplättchen auf. $2 \mathrm{~m}$ oberhalb der Basis des 


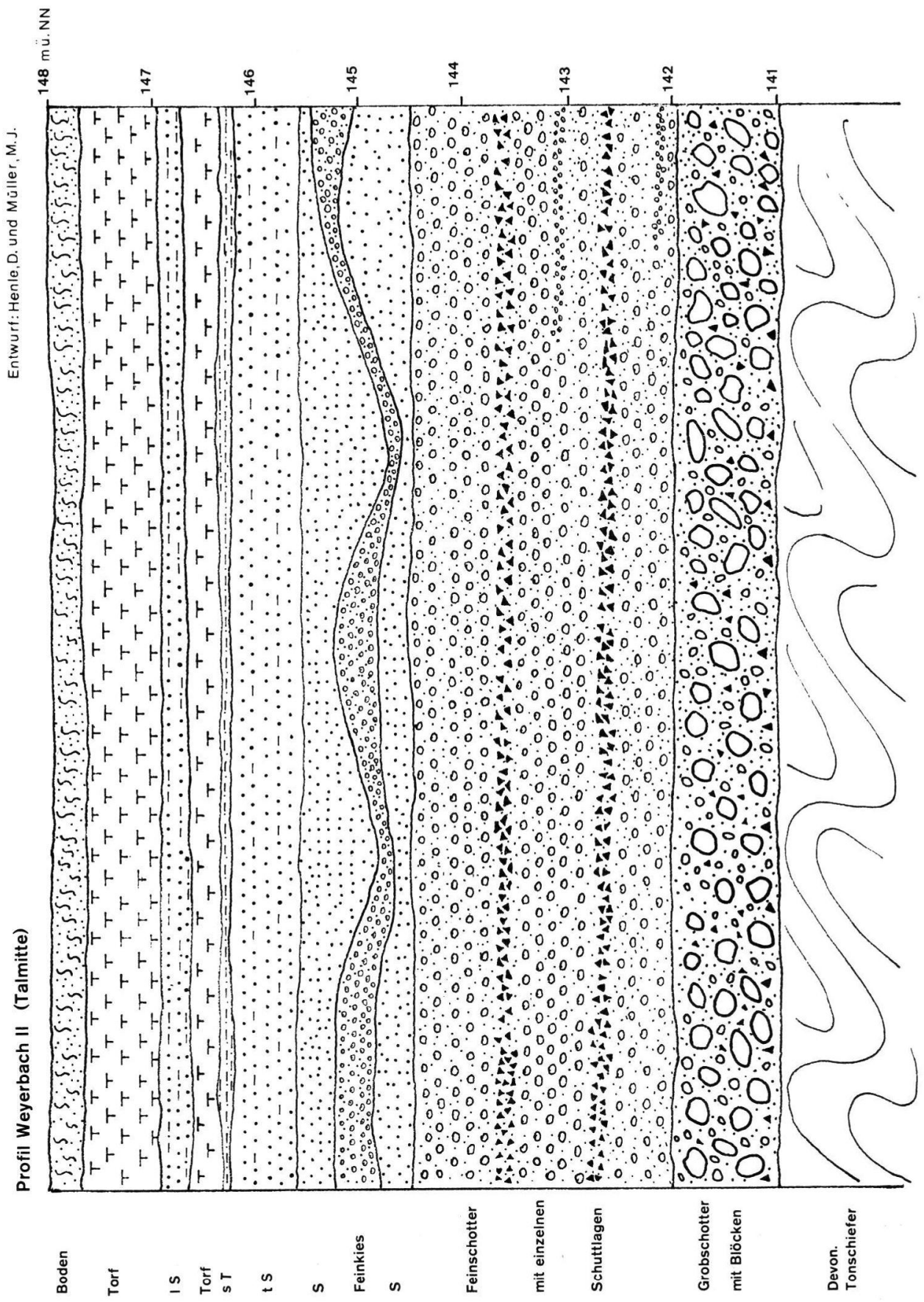

Abb. 4: Saarmäander Ayl-Wawern-Biebelhausen, Profil Weyerbach II (Talmitte). 
Tab. 3: Bodenchemische Eigenschaften der Böden

\begin{tabular}{|c|c|c|c|c|c|}
\hline $\begin{array}{l}\text { Tiefe } \\
\text { (m) }\end{array}$ & $\underset{\mathrm{CaCl}_{2}}{\mathrm{pH}}$ & $\underset{\%}{\mathrm{C}}$ & $\begin{array}{c}\mathrm{Fe}_{\mathrm{o}} \\
0 \% 0\end{array}$ & $\begin{array}{c}\mathrm{Fe}_{\mathrm{d}} \\
0 \%\end{array}$ & $\underset{0 \%}{\mathrm{P}_{2} \mathrm{O}_{5}}$ \\
\hline \multicolumn{6}{|c|}{ rezenter Boden } \\
\hline $\begin{array}{l}0,0,2 \\
0,2=0,5 \\
0,5=0,85 \\
0,85-1,1 \\
1,1-1,60\end{array}$ & $\begin{array}{l}4,8 \\
5,3 \\
5,4 \\
5,5 \\
5,0\end{array}$ & $\begin{array}{l}0,90 \\
0,40 \\
0,26 \\
0,13 \\
0,15\end{array}$ & $\begin{array}{l}0,24 \\
0,28 \\
0,24 \\
0,23 \\
0,15\end{array}$ & $\begin{array}{l}0,61 \\
0,74 \\
0,70 \\
0,54 \\
0,70\end{array}$ & $\begin{array}{l}0,14 \\
0,14 \\
0,11 \\
0,06 \\
0,07\end{array}$ \\
\hline \multicolumn{6}{|c|}{ oberer fossiler Boden } \\
\hline $\begin{array}{l}6,00-6,25 \\
6,25-6,55 \\
7,8-7,9 \\
7,9-8,0\end{array}$ & $\begin{array}{l}5,7 \\
5,8 \\
5,6 \\
5,6\end{array}$ & $\begin{array}{l}0,36 \\
0,36 \\
0,05 \\
0,10\end{array}$ & $\begin{array}{l}0,12 \\
0,12 \\
0,12 \\
0,13\end{array}$ & $\begin{array}{l}0,86 \\
0,71 \\
0,52 \\
0,76\end{array}$ & $\begin{array}{l}0,11 \\
0,07 \\
0,06 \\
0,07\end{array}$ \\
\hline \multicolumn{6}{|c|}{ mittlerer fossiler Boden } \\
\hline $\begin{array}{l}8,4-8,8 \\
8,8-9,25 \\
9,25-9,6 \\
9,6-9,9\end{array}$ & $\begin{array}{l}5,6 \\
5,3 \\
5,3 \\
5,3\end{array}$ & $\begin{array}{l}0,50 \\
0,48 \\
0,16 \\
0,17\end{array}$ & $\begin{array}{l}0,17 \\
0,18 \\
0,11 \\
0,08\end{array}$ & $\begin{array}{l}0,67 \\
0,64 \\
0,52 \\
0,56\end{array}$ & $\begin{array}{l}0,08 \\
0,06 \\
0,05 \\
0,04\end{array}$ \\
\hline \multicolumn{6}{|c|}{ unterer fossiler Boden } \\
\hline $\begin{array}{c}9,9-10,2 \\
10,25-11,15 \\
11,15-12,15\end{array}$ & $\begin{array}{l}5,3 \\
5,1 \\
5,5\end{array}$ & $\begin{array}{l}0,25 \\
0,16 \\
0,15\end{array}$ & $\begin{array}{l}0,11 \\
0,05 \\
0,09\end{array}$ & $\begin{array}{l}0,57 \\
0,47 \\
0,58\end{array}$ & $\begin{array}{l}0,06 \\
0,06 \\
0,05\end{array}$ \\
\hline
\end{tabular}

Tones, die teilweise von anstehenden devonischen Schiefern teilweise von Feinschotter gebildet wird, wurde ein Kiefernholz gefunden ${ }^{2}$ ). Die Altersbestimmung dieses Holzstückes ergab ein ${ }^{14} \mathrm{C}$-Alter von $15800 \pm 300$ Jahren $^{3}$ ).

Aus dem Ton wurden im Abstand von $20 \mathrm{~cm} 28$ Proben genommen. 13 davon konnten pollenanalytisch ausgewertet werden (Abb. 5). Auffallend ist ein zum Teil hoher Anteil präquartärer Sporomorphen, der an der Basis des Profils besonders hoch ist.

Dieser Anteil an umgelagerten Sporomorphen ist wohl durch die Herkunft des Sedimentes bedingt.

Die Kurvenverläufe der einzelnen Taxa lassen im wesentlichen keine großen Schwankungen erkennen, so daß eine Gliederung des Profils anhand des Baumpollens nahezu ausgeschlossen ist. Nur an der Profilbasis fallen höhere Pinusanteile auf, bevor die Pinuskurve auf ungefähr $30 \%$ absinkt. Im Gegensatz zu den präquartären Sporomorphen sind Pinus und der übrige Pollen gut erhalten. Unter dem Baumpollen kommt noch Fichte, Birke, Weide, Pappel und Erle vor, wobei die einzelnen Taxa nur geringe Werte aufweisen. Es herrschte eine typenreiche Kräuterflora vor, die partiell von Artemisia dominiert wird. Auch Gramineae spielen teilweise eine größere Rolle. Man könnte von einer kräuterreichen Artemisiensteppe sprechen, wobei sich der Kiefernanteil am ehesten mit Fernflug erklären ließe. Paläoklimatisch läßt sich dieses Profil wohl nur als unter hochglazialen Bedingungen entstanden interpretieren. Möglicherweise deutet

2) Für die Holzbestimmungen danken wir Herrn Obermuseumsrat Ennst Hollstein, Trier.

3) Für die Altersbestimmungen bedanken wir uns bei Prof. Dr. Scharpenseel, Hamburg. 


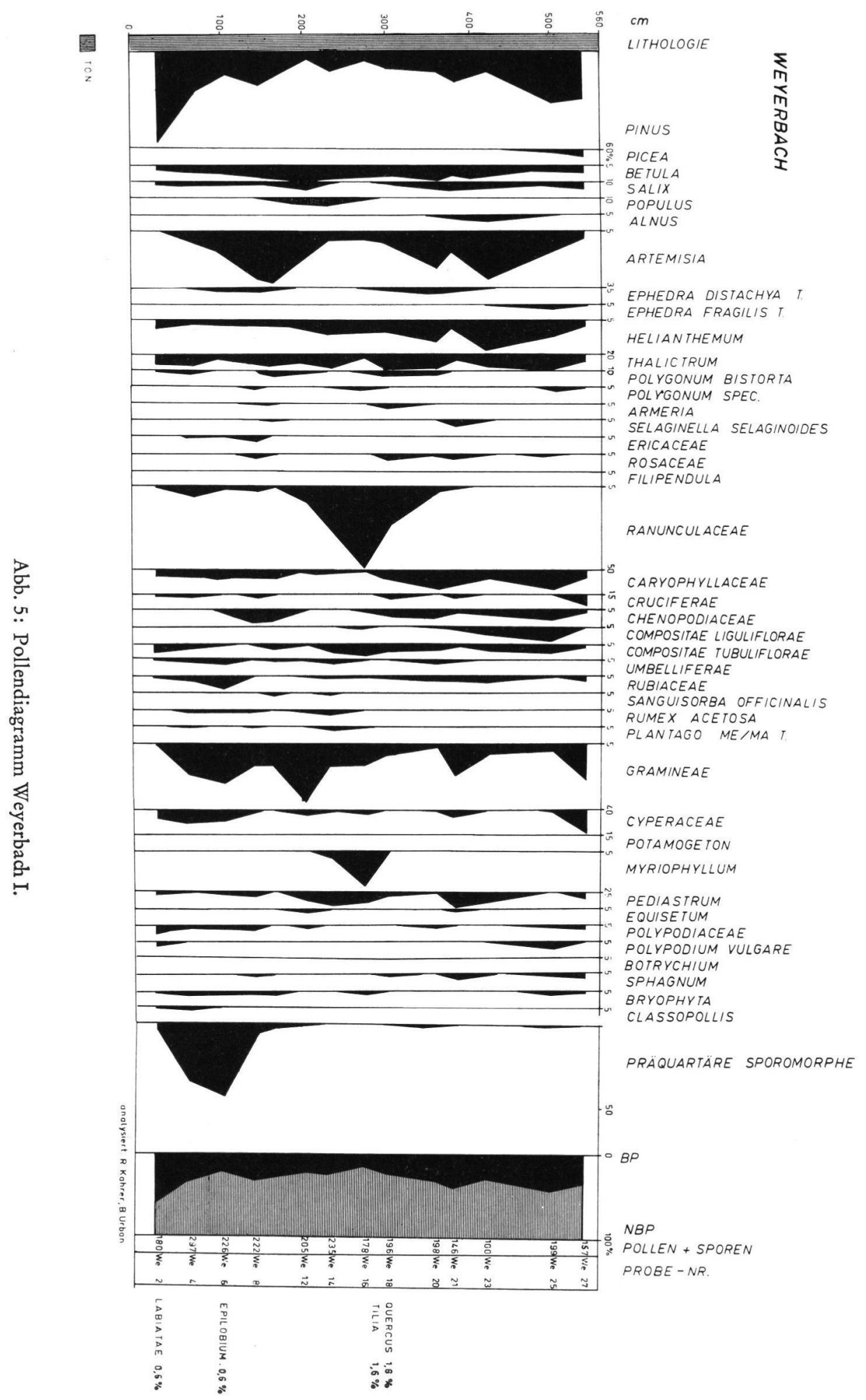


Tab. 4: Stratigraphische Zuordnung

Profilaufbau

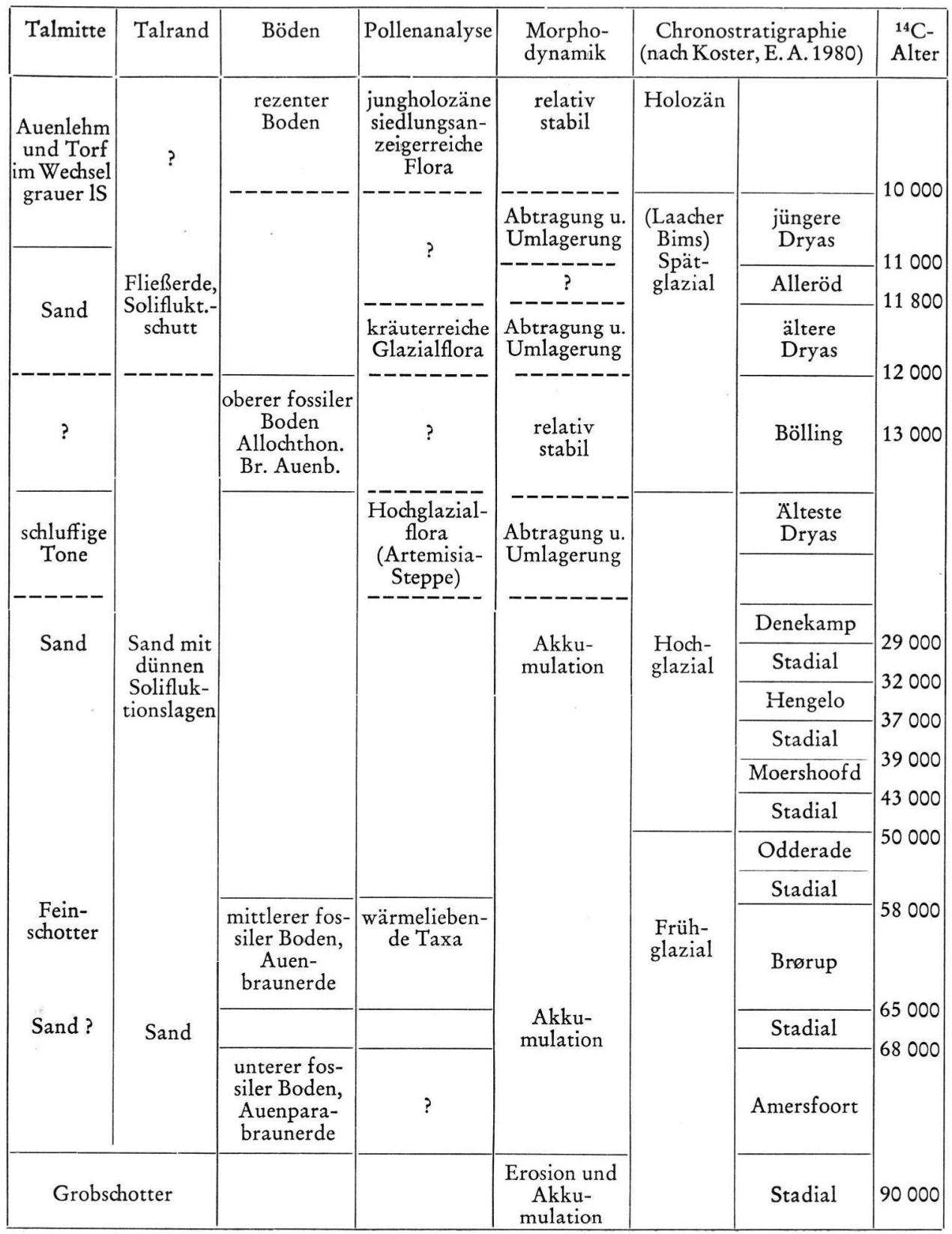




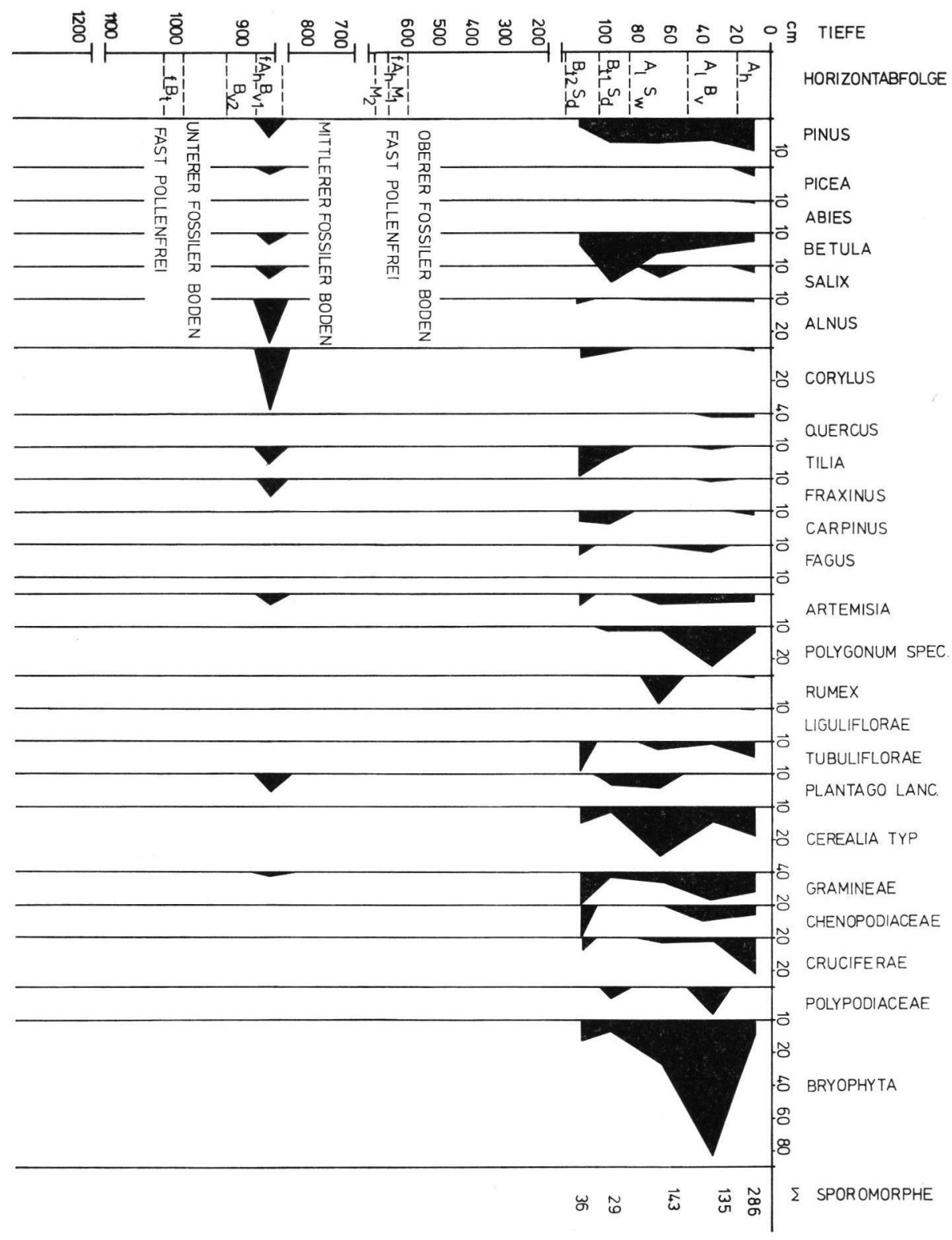

Abb. 6: Pollendiagramm Saarkanal.

sich an der Basis das Ende eines Interstadials an. Für Umlagerungen interstadialen oder interglazialen Materials gibt es keinerlei Hinweise. Die gleichförmigen Kurvenverläufe deuten ein ruhiges Ablagerungsmilieu an.

Die Ergebnisse der Pollenanalyse passen damit sehr gut zu der Altersbestimmung des Holzes. Es kann also angenommen werden, daß die Tone im Hochglazial sedimentiert wurden. 
Uber dem Ton, aber auch über dem Feinschotter liegen braune geschichtete Sande, die in der Talmitte $3 \mathrm{~m}$ Mächtigkeit erreichen. Sie werden zeitlich den bis $\mathrm{zu} 6 \mathrm{~m}$ mächtigen Sanden am Rand des Tales, in denen sich der obere fossile Boden befindet, gleichgestellt. Hangaufwärts verzahnen sie sich mit Fließerdezungen. Im oberen Abschnitt des Hanges werden sie von einem bis zu $10 \mathrm{~m}$ mächtigen Solifluktionsschutt unterlagert. Über den Sanden liegt die schon erwähnte Fließerde aus der Alteren Dryas.

In der Talmitte, in der nach dem Mäanderdurchbruch der Weyerbach sein schmales Bett geschaffen hat, werden die spätweichselzeitlichen Sande von einem sandigen Lehm und einer $1 \mathrm{~m}$ bis $3 \mathrm{~m}$ mächtigen Torfschicht bedeckt. Ein Erlenholz an der Basis des Torfes im Lehm hat ein ${ }^{14} \mathrm{C}$-Alter von $2800 \pm 200$ Jahren. Die Pollenanalyse ergab, abgesehen von der Erle, einen geringen Baumpollenanteil (Abb. 6).

In Verbindung mit Wasserpflanzen und feuchtigkeitsliebenden Taxa kann als Ablagerungsmilieu ein schwach bewegtes Gewässer, bzw. ein Gewässerrand mit lokalem Erlenbruch angenommen werden. Die Kräuterflora ist sehr typenreich; es befindet sich darunter auch Pollen von Kräuterpflanzen und Siedlungsanzeigern wie etwa: Wegerich (Plantago lanceolata, Plantago major/media) oder auch Knöterich (Polygonum bistorta) und Artemisia. Getreidepollen tritt in Spuren auf. Unter dem Baumpollen haben, von der lokalen Dominanz der Erle abgesehen, Eiche und Kiefer, im jüngsten Abschnitt auch die Hainbuche, die höchsten Werte (um 10\%). Vereinzelt tritt Buchen-, Fichten- und Tannenpollen auf. Die Pollenspektren deuten insgesamt gesehen auf lichte Bewaldung der näheren Umgebung hin; für den Bildungszeitraum ist eine starke Nutzung des Untersuchungsgebietes durch den Menschen anzunehmen.

\section{2. Überlegungen zur Morphodynamik}

Aufgrund der Untersuchungsergebnisse, die u. a. zwei absolute Datierungen im Sediment erbrachten, kann man sich die Entwicklung des Saarmäanders von Ayl-WawernBiebelhausen folgendermaßen vorstellen:

Während des Weichselfrühglazials hat die Saar sich kräftig eingeschnitten und einen Grobschotter hinterlassen. Er ist nicht überall im Flußbett erhalten, erreicht aber in der Regel in der Flußmitte eine Mächtigkeit von $2 \mathrm{~m}$. Ob dieser Schotterkörper einmal mächtiger gewesen ist, läßt sich nicht sagen.

Schon im Frühglazial hat die Saar im großen und ganzen das endgültige Erosionsniveau der Niederterrasse erreicht. Über dem Grobschotter ist entweder gegen Ende des ersten Stadials oder zu Beginn des Amersfoort-Interstadials ein mächtiges Sandpaket akkumuliert worden, auf dem sich eine Auen-Parabraunerde entwickelt hat. Diese Sande sind im folgenden Stadial wieder ausgeräumt worden und nur an wenigen günstigen Stellen erhalten geblieben. Daraufhin wurde ein Feinschotterkörper von etwa 2,5 m Mächtigkeit abgelagert. Gegen Ende dieses Stadials oder zu Beginn des BrørupInterstadials schüttete die Saar wieder mächtige Sande auf, auf denen sich der mittlere Boden entwickelte. Gegen Ende des Frühglazials oder zu Beginn des Hochglazials hat der Mäanderdurchbruch stattgefunden, denn über dem Sand treten keine grobklastischen Sedimente mehr auf, sondern nur noch tonig-schluffige.

Auf jeden Fall aber war der Durchbruch schon vor dem Spätglazial vollzogen. Die an verschiedenen Stellen direkt auf dem Grobschotter oder sogar über dem Tonschiefer liegenden Tone lassen sich nur erklären als Ablagerungen in einem Altarm. Der abgeschnittene Mäander hat bei größeren Hochwässern sozusagen als Entlastungsrinne gedient. In den Schottern haben sich Kolke gebildet, und diese sind dann als kleine 
Tümpel langsam mit Feinsedimenten aufgefüllt worden. Das ${ }^{14} \mathrm{C}-$ Alter nahe der Basis der Tone ist $15800 \pm 300$ Jahre, und das Pollenspektrum weist von unten bis oben kaltzeitliche Verhältnisse auf. Mächtige Hochwässer müssen diesen Altarm aber auch noch gegen Ende des Hochglazials und zu Beginn des Spätglazials durchflossen haben, denn es liegen über dem Ton noch Saarsande, in denen sich der Böllingboden entwickelt hat. Sie sind bevorzugt in einer vom Hang herunterziehenden Delle, an einem Prallhang der frühglazialen Saar, sedimentiert worden, in der günstige Sedimentationsbedingungen herrschten. Eine gewisse Schwierigkeit bereitet die Erklärung der außerordentlich hohen Lage dieser Sande. Immerhin liegt ihre Oberkante etwa $20 \mathrm{~m}$ über der Flußsohle und noch rund $15 \mathrm{~m}$ über den frühglazialen Schottern. Das höchste Hochwasser der Saar erreichte 1947 etwa $6 \mathrm{~m}$ über normalem Wasserstand. Für das Spätglazial müssen wir erheblich höhere Hochwässer annehmen.

\section{Profilbeschreibung der Böden}

\section{Rezenter B od en: Parabraunerde aus lößhaltiger Fließerde über Terrassensand}
$\mathrm{A}_{\mathrm{b}} \quad$ O- $20 \mathrm{~cm}$, schwarzbrauner (7,5 YR 4/4), etwas Schiefergrus enthaltender $1 \mathrm{~S}$, mittelhumos, krümelig bis plattig, gut durchwurzelt, allmählicher Übergang
$A_{l} B_{v} \quad 20-50 \mathrm{~cm}$, brauner (7,5 YR 4/6), viel Schiefergrus enthaltender sL, lößreich, subpolyedrisch, schwach durchwurzelt, einzelne Wurmgänge, unschar- fer Ubergang
$\mathrm{A}_{\mathrm{l}} \mathrm{S}_{\mathrm{vv}} \quad 50-80 \mathrm{~cm}$, brauner (7,5 YR 4/6), von grauen (10 YR 6/4) Reduktionsflecken durchsetzter, lößreicher sL, subpolyedrisch-polyedrisch, schwach durch- wurzelt, einzelne Wurmgänge, zahlreiche Konkretionen, allmählicher Ubergang
II $\mathrm{B}_{\mathrm{t} 1} \mathrm{~S}_{\mathrm{b}} \quad 85-110 \mathrm{~cm}$, rotbrauner (5 YR 4/8), von grauen (10 YR 6/4) Reduktionsflecken und -spalten durchsetzter $1 S$ aus Lößsolifluktionsmaterial und einge- mischtem Terrassensand, polyedrisch-prismatisch, einzelne Wurmgänge, welliger, deutlicher Ubergang
II $\mathrm{B}_{\mathrm{t} 2} \mathrm{~S}_{\mathrm{b}} \quad 110-160 \mathrm{~cm}$, rotbrauner (5 YR 4/6) sL, kohärent $160 \mathrm{~cm}$, rotbrauner (5 YR 4/8) Terrassensand

zwischen 160 und $600 \mathrm{~cm}$ brauner lehmiger Sand

\section{Oberer fossiler Boden: Allochthoner brauner Auenboden aus Hochflutsand}
$f \mathrm{~A}_{\mathrm{h}} \mathrm{M}_{1} \quad 600-625 \mathrm{~cm}$, hellrotbrauner (5 YR 5/6) 1S, porös, locker, kohärent bis sub- polyedrisch
$\mathrm{f} \mathrm{M}_{2} \quad 625-655 \mathrm{~cm}$, rotbrauner (5 YR 4/6) 1S, mittelfest, subpolyedrisch, zahlreiche Wurm- gänge, allmählicher Übergang in braunen Horizont mit Eisen und Ton angereicherten Bändern, diese dicht, fest, tonreicher, dunkelrotbraun (5 YR 3/6) Sand zwischen Bändern (5 YR 5/6), im gesamten Profil Schieferstückchen, im unteren Teil (unterhalb der Bänder) solifluidal $780-790 \mathrm{~cm}$, heller $\mathrm{tS}$ eingeregelte feine Schichten aus Schieferplättchen
$790-800 \mathrm{~cm}$, rotbraunes Band aus $\mathrm{tS}$
$800-840 \mathrm{~cm}$, heller Sand, schwach Fe-Mn-gefleckt




\section{Mittlerer fossiler Boden: Auenbraunerde aus Hochflutlehm}

\begin{tabular}{|c|c|}
\hline $\mathrm{fA}_{\mathrm{h}} \mathrm{B}_{\mathrm{v}} 1$ & $\begin{array}{c}\text { 840-880 cm, hellrotbrauner (5 YR 4/6) } \overline{\mathrm{IS}} \text {, plattig (Solifluktion), eingereg. Schiefer- } \\
\text { plättchen, keine Wurmröhren, scharfer, gerader Úbergang }\end{array}$ \\
\hline $\mathrm{fA}_{\mathrm{h}} \mathrm{B}_{\mathrm{v} 2}$ & $\begin{array}{l}\text { 880-925 cm, rotbrauner ( } 5 \text { YR 4/8) } \overline{\mathrm{IS}} \text {, festes Subpolyedergefüge, zahlreiche Re- } \\
\text { genwurmgänge, einige Schieferstückchen, allmählicher Ubergang }\end{array}$ \\
\hline $\mathrm{fB}_{\mathrm{v}} \mathrm{C}$ & $\begin{array}{c}\text { 925-960 cm, hellrotbrauner ( } 5 \text { YR 5/6) IS, kohärent, locker, keine Bänderung, } \\
\text { lediglich allmählicher Ubergang von rot in hellrotbraun }\end{array}$ \\
\hline C & $960-990 \mathrm{~cm}$, mattrotbrauner (5 YR 5/4) ÍS \\
\hline
\end{tabular}

Unterer fossiler Boden: stark erodierte Auen-Parabraunerde

A-Horizonte fehlen

$\mathrm{fB}_{\mathrm{t}} \quad 990-1020 \mathrm{~cm}$, dunkelrotbrauner (5 YR 3/6), $\overline{\mathrm{IS}}$, einige Wurmröhren, sehr heterogen, einige helle Sandlinsen wechseln mit rotbraunen Partien (in Auflösung begriffener $B_{t}$ ), sehr starker kleinräumiger Wechsel von hellem Sand zu rotbraunem 1S, polyedrisch, sehr schwach Fe-Mn-gefleckt

C $\quad 1020-1115 \mathrm{~cm}$, mattrotbrauner (5 YR 5/4) Sand

$1115-1215 \mathrm{~cm}$, rotbrauner $\mathrm{tS}$ in fleckweisem Wechsel mit hellem Sand (Eisen- und Tonanreicherungen).

\section{Schriftenverzeichnis}

Fischer, F. (1957): Beiträge zur Morphologie des Flußsystems der Saar. - Universität des Saarlandes, Arbeiten aus dem Geographischen Institut, 2: 5-92; Saarbrücken.

Grebe, H. (1890): Über Tertiär-Vorkommen zu beiden Seiten des Rheins zwischen Bingen und Lahnstein und Weiteres über Talbildung am Rhein, an der Saar und Mosel. - Jb. kgl. preuß. geolog. L.-Anst. für 1889: 99-123; Berlin.

HenRich, H. W. (1958): Der Schwermineralgehalt der Terrassen im Unterlauf der Saar. - Ann. Univ. Saraviensis, Naturwiss., VII: $118-145$; Saarbrücken.

Koster, E. A. (1980): De Indeling van het kwartair van Nederland. Toelichting big de stratigrafische Tabellen van het Pleistoceen en het Holoceen. - Geografisch Tijdschrift, Nieuve reeks XIV, afl. 4. (Beilage).

Mathias, K. (1936): Morphologie des Saartals zwischen Saarbrücken und der Saarmündung. -Decheniana. Verhandl. naturhist. Ver. Rheinland u. Westfalen, 93: 1-112; Bonn.

- (1952): Die Entwicklung der Talmäander im Bereich der unteren Saar. - Ann. Universitatis Saraviensis, Naturwiss. 4: 355-369; Saarbrücken.

Müller, M. J. (1978): Die Entwicklung der Oberflächenformen im unteren Saartal, im Moseltal und in der "Wittlicher Senke". - In: Trier und Umgebung. Sammlung Geographischer Führer 11: 10-18; Berlin, Stuttgart (Bornträger).

- (1979): Die Umlaufberge im unteren Saartal. - In: Beiträge zur trierischen Landeskunde: 353-361; Trier.

- (1983): Geomorphologische Kartierung der Karte 1:25000, Blatt 6305 Saarburg; mit Erläuterungsband. - Berlin. - [Im Druck].

Ricken, W. \& Meyer, B. (1982): Lößbürtige Böden des Jung-Quartärs zwischen Harz und Leine. — Mitt. Dtsch. Bodenkundl. Ges., 34: 249-268; Göttingen.

SCHRöDER, D. (1979): Bodenentwicklung in spätpleistozänen Hochflutlehmen des Niederrheins. Habilitationsschrift, Bonn, 296 S.; Bonn.

Stephan, S. (1981): Zur Mikromorphologie der Tonverlagerung im Alluvium des Niederrheins. In: Sonderveröff. Geograph. Inst. Univ. Köln, 41: 243-247; Köln.

ZANDSTRA, K. J. (1954): Die jungquartäre morphologische Entwicklung des Saartals. - Erdkunde, VIII: 276-285; Bonn.

ZöLler, L. (1980): Über Hangschuttbildung, Plateaulehme und junge Erosion im „Hochwald“, westlicher Hunsrück, Rheinisches Schiefergebirge. - Catena, 7: 153-167; Braunschweig. 